\title{
Secure role based access control systems using aspect-orientation designing
}

\begin{abstract}
Security system designs are required to be flexible enough to support multiple policies. A security policy model always develops; accordingly, the design of a security system using that policy model should reflect the changes. Using role-based access control (RBAC) as an example, currently it supports role hierarchy, static separation of duty relations, and dynamic separation of duty relations. As research on RBAC progresses, more concerns have been and will be covered. So the model hierarchy of RBAC is quickly becoming more and more complicated, which requires that the security system supporting RBAC be flexible and extensible. To address this issue at the design level, we propose an aspect-oriented approach to designing flexible and extensible security systems. This paper illustrates the approach through a case study, which is part of a design for CORBA access control (AC) supporting RBAC models.
\end{abstract}

Keyword: Security system designs; Security policy model; Role-based access control (RBAC) 